\title{
Trench-Assisted All-Solid Large-Mode-Area Fiber for Robust Single-Mode Operation
}

\author{
Jayanta K. Sahu,* Deepak Jain, and Yongmin Jung \\ Optoelectronics research center, University of Southampton, UK \\ email: jks@orc.soton.ac.uk
}

\begin{abstract}
In this talk, we discuss all-solid and easily realizable trench-assisted fibers that show tremendous potential for addressing challenges faced by current fiber designs for power scaling. OCIS codes: (000.0000) General; (000.0000) General [8-pt. type. For codes, see www.opticsinfobase.org/submit/ocis.]
\end{abstract}

\section{Introduction}

Optical fibers have made tremendous contribution to mankind in last decades by revolutionizing the field of high power fiber lasers [1]. In order to fulfill the increasing demand of high output power from a laser, there is a need for optical fiber which can provide large effective area $\left(\mathrm{A}_{\mathrm{eff}}\right)$ of the fundamental mode $(\mathrm{FM})$ to avoid non-linear effects while simultaneously offering high suppression to higher order modes (HOMs) to preserve beam quality [2]. Unfortunately, step index fiber is unable to offer a balance between increasing $A_{\text {eff }}$ of the FM and suppression of the HOMs. Over the past years, several fiber designs have been proposed such as photonic crystal fiber (PCF), Leakage channel fiber (LCF), Photonic bandgap fibers etc. Unfortunately, the improvement in terms of offering large $A_{\text {eff }}$, while preserving single mode operation comes on the cost of dramatic increase in fiber fabrication complexities, difficult fiber handling (such as difficult cleaving and splicing due to air holes) etc. Moreover, in most of the fiber designs, the critical requirement of refractive index matching of core, which is doped with rare-earth ions, to that of cladding has been a constant challenge for fabricators with increasing demand of $\mathrm{A}_{\text {eff }}$ in fiber.

In order to address these issues, we have recently proposed all-solid single trench fiber (STF) having cylindrical symmetry and higher refractive index of core as of cladding for bend configuration that is needed for a compact laser device [3-5]. Our optimized modified chemical vapour deposition (MCVD) process in conjunction with solution doping technique has shown the suitability of STF design for mass-scale production. On the other hand, we proposed Multi-trench fibers (MTF) having all-solid cylindrical symmetrical design for rod-type configuration [6-8]. Rod-type fibers can offer ultra-large $\mathrm{A}_{\text {eff }}$ (few $1000 \mu \mathrm{m}^{2}$ ), however they need to keep straight and require the core refractive index same as of cladding. Nevertheless, both of these fiber designs have shown tremendous potential for fiber laser applications. In this paper, we review our progress towards MTF and STF for high power fiber laser applications.

\section{Single trench fiber}

Fig. 1(a) shows the schematic of refractive index profile (RIP) of our proposed single trench fiber (STF) and Fig. 1(b) shows the 2D cross-section of the STF. STF offers high delocalization and loss to the HOMs, thanks to the resonant coupling between core and ring modes. Numerical analysis shows that an $\mathrm{A}_{\mathrm{eff}}$ as large as $1,500 \mu \mathrm{m}^{2}$ can be achieved at $20 \mathrm{~cm}$ bend radius by exploiting the high loss of HOMs (larger than $10 \mathrm{~dB} / \mathrm{m}$ ) or low power fraction of HOMs in core $(<50 \%)$. On the other hand, the FM loss remains lower than $0.1 \mathrm{~dB} / \mathrm{m}$ and high power fraction in core $(>80 \%)$. The detail of numerical analysis is reported in [5].

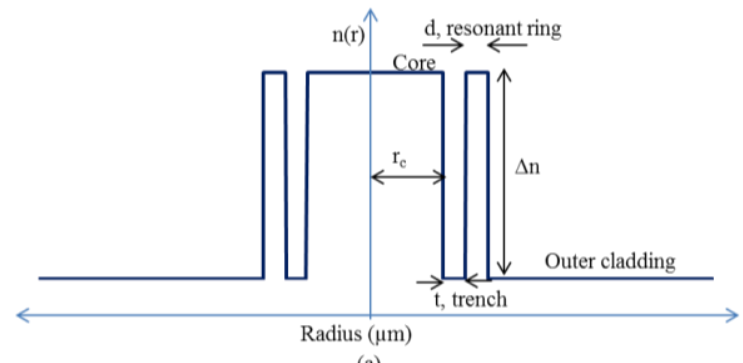

(a)

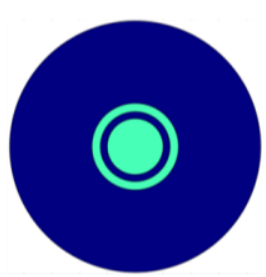

(b)

Fig. 1 (a) Schematic of refractive index profile (RIP) of STF and Fig. 1(b) shows the 2-D cross-section of STF. 
The STF is an all-solid structure and refractive index of core is higher than of cladding as shown in Fig. 1(a). Due to these two features, it has been fabricated by conventional MCVD process in conjunction with rod-in-tube process as reported in our previous studies [3-5]. We have fabricated three Yb-doped STFs having different core diameters of $20 \mu \mathrm{m}, 30 \mu \mathrm{m}$, and $40 \mu \mathrm{m}$. Characterizations for these fibers ensure a Gaussian output. The details of experimental

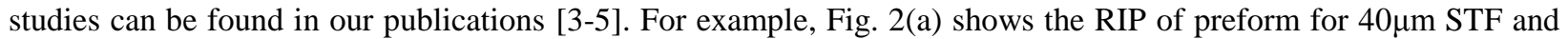
Fig. 2(b) shows the experimental set-up used for characterizations. Fig. 2(c) shows the output beam with respect to single mode input beam, on the other hand Fig. 2(d) and Fig. 2(e) shows the output beam with respect to multimoded beam. These measurements ensure a robust single mode operation and fiber also ensure good laser efficiency $(>75 \%)$ at wavelength $\sim 1040 \mathrm{~nm}$.

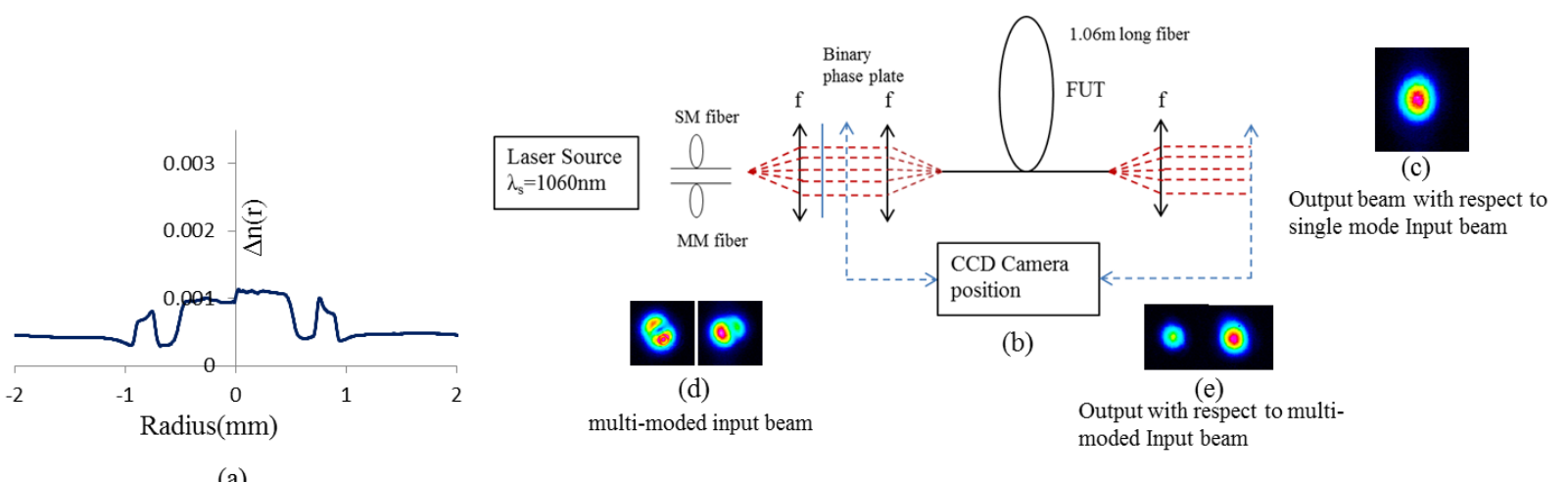

Fig. 2(a) RIP of preform (b) Experimental set-up used for characterizations(c) Output beam profile w.r.t different single mode input (d) input multi-moded launched and (e) output beam profile with respect to these mixed $\left(\mathrm{LP}_{01}+\mathrm{LP}_{11}\right)$ mode.

\section{Multi trench fiber}

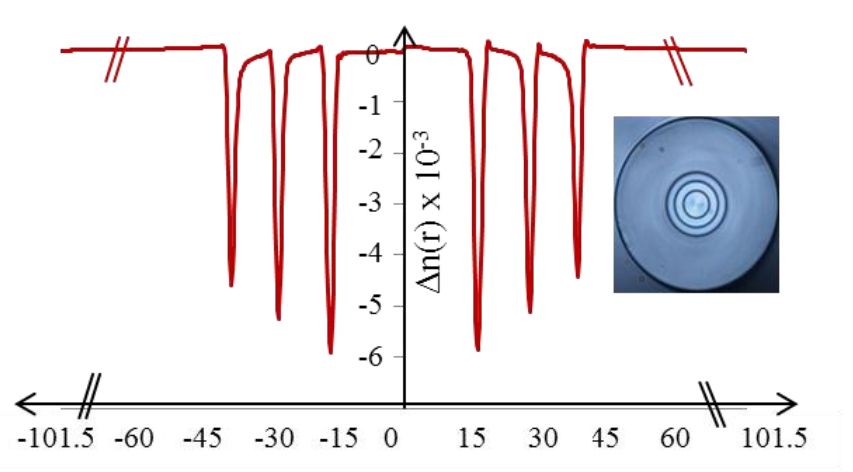

Fiber radius $(\mu \mathrm{m})$

(a)
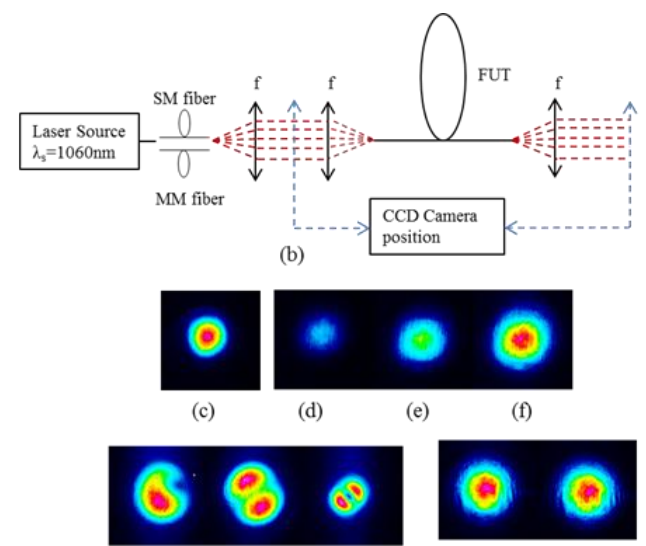

(g)

Fig. 3 (a) The fabricated refractive index profile of 30um MTF. Inset shows the microscope image of fiber cross-section. (b) experimental set-up used for singlemode verification (c) the output profile for optimum input launching (d-f) output profile for offset input launching conditions. (g) various input launch modes (h) output profiles with respect to multimode input launch. 
Figure 3 (a) shows the RIP of the fabricated $30 \mu \mathrm{m}$ MTF for use in bend configuration. Numerical simulations show that a $30 \mu \mathrm{m}$ core diameter MTF with $\mathrm{d}=8 \mu \mathrm{m}, \mathrm{t}=2 \mu \mathrm{m}$, and $\Delta \mathrm{n}=-0.005$, provides an effective area $\left(\mathrm{A}_{\text {eff }}\right)$ of the FM larger than $407 \mu \mathrm{m}^{2}$ in $28-50 \mathrm{~cm}$ bend diameter range at $1060 \mathrm{~nm}$. Figure 3(b) shows the experimental set-up used for single mode behaviour of fiber. For various off-set and multimode launching, fiber ensures a single mode operation. We also compared fibers with high and low-index coating to study the impact of polymer coating on single-mode behaviour. $S^{2}$ measurement ensures effective single-mode behaviour in both high and low-index polymer coated fibers. The detailed study is reported in references [6-8]. On the other hand, Fig. 4(a) shows the fabricated RIP of a $90 \mu \mathrm{m}$ core MTF. Again this fiber was fabricated using MCVD in conjunction with rod-in-tube technique. Characterization ensures a Gaussian output beam as shown in Fig. 4(b) and 4(c) [8].

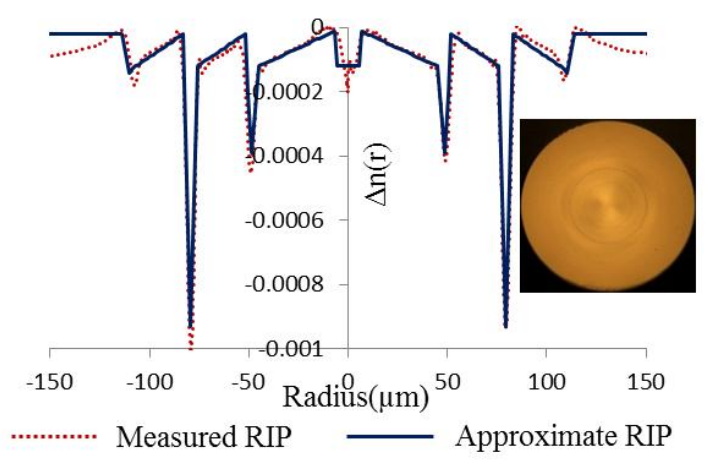

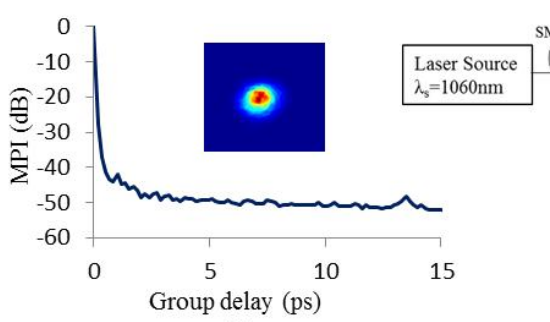

(b)

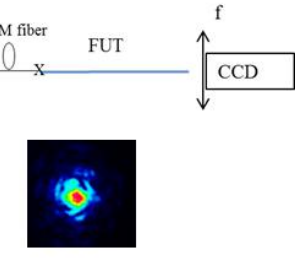

(c)

(a)

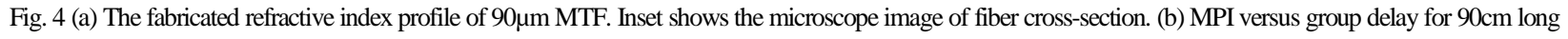
rod-type fiber. Inset shows the obtained FM image from $\mathrm{S}^{2}$ measurement. (b) Output image captured by CCD camera using set up shown here

\section{Conclusion}

We have demonstrated novel large-mode area fibers for high power fiber applications. Our proposed designs can offer $\mathrm{A}_{\text {eff }}$ of $\sim 1,500$ and $\sim 10,000 \mu \mathrm{m}^{2}$ at wavelength $\sim 1060 \mathrm{~nm}$ in a compact and rod-type fiber laser configuration respectively. The trench-assisted fibers are suitable for large scale manufacturing, and furthermore being an allsolid and cylindrical structure they ensure easy cleaving and splicing.

\section{References}

[1] C. Jauregui et. al., "High-power fiber lasers," Review, Nature Photonics, vol. 7, 861-867, 2013.

[2] G. P. Agrawal et. al., "Nonlinear Fiber optics", $4^{\text {th }}$ edition, Academic Press, 2007.

[3] D. Jain et. al., "Large mode area multi-trench fiber with delocalization of higher order modes," Invited, IEEE JSTQE $20,0902909,2014$.

[4] D. Jain et. al., "First demonstration of single trench fiber for delocalization of higher order modes," Invited, SF1N.1, CLEO, San Jose, 2014.

[5] D. Jain et. al., "Extending single mode performance of all-solid large-mode-area single trench fiber," Opt. Exp., submitted (2014).

[6] D. Jain, et. al., "Mode area scaling with Multi-trench rod-type fibers," Opt. Exp. 21, 1448-1455, 2013.

[7] D. Jain et. al., "Bending performance of large mode area multi-trench fibers," Opt. Exp. 21, 26663-26670, 2013.

[8] D. Jain et. al., "Robust single-mode all-solid multi-trench fiber with large effective mode area," Opt. Lett. 39, $5200-5203$ (2014). 